\title{
Utilidad de la monitorización terapéutica de infliximab en el manejo de la enfermedad inflamatoria intestinal
}

\author{
RODRIGO QUERA ${ }^{1,2}$, MAURICIO MORENO ${ }^{3, \mathrm{c}}$, \\ DANIELA SIMIAN ${ }^{1,4, a}$, PATRICIO IBÁÑEZ ${ }^{1,2}$, JAIME LUBASCHER $^{1,2}$, \\ CAROLINA FIGUEROA ${ }^{1,2}$, LILIAN FLORES ${ }^{1, a}$, UDO KRONBERG ${ }^{1,5}$, \\ GONZALO PIZARRO ${ }^{1,2,6}$, DANIELA FLUXÁ ${ }^{2 b}$
}

\section{Usefulness of therapeutic monitoring of infliximab in the treatment of inflammatory bowel disease}

Background: Primary non-response and secondary loss of response (LOR) are significant problems of biological therapy for inflammatory bowel disease (IBD). Therapeutic drug monitoring (TDM) in IBD patients receiving these drugs can improve outcomes. Aim: To measure serum infliximab levels and anti-infliximab antibodies (ATI) in patients with IBD post-induction phase and during maintenance therapy assessing the clinical course of IBD. Patients and Methods: Prospective study of IBD patients receiving infliximab between July 2016-May 2017. Group-A included patients who received induction therapy while Group-B included patients who were in maintenance therapy. TDM was performed in serum samples collected at weeks-14 and 30 in Group-A and before the infliximab maintenance dose in Group-B. Clinical scores, fecal calprotectin and endoscopic score were also evaluated. Results: Of 14 patients in Group-A, 57\% achieved endoscopic response. Median serum infliximab concentrations at week-14 and 30 were $2.65 \mathrm{AU} /$ $m L$ (0.23-32.58) and $2.3 \mathrm{AU} / \mathrm{mL}$ (0.3-16.8), respectively. Patients with mucosal healing had non-significantly higher median infliximab concentrations at week14, as compared to week 30 (median 3.2 vs $2.2 \mathrm{AU} / \mathrm{ml}$, respectively, p 0.6). ATI $>10 \mathrm{ug} / \mathrm{mL}$ were found in one and seven patients at week-14 and 30, respectively. At 52 weeks of follow-up, four patients (31\%) had LOR. Group-B included 36 patients, 33\% had LOR. Median serum concentrations of infliximab were 1.4 AU/mL (0.27-7.03). No significant differences in serum infliximab concentration were observed between patients in remission and those with inflammatory activity. Seventeen patients had ATI $>10 \mathrm{ug} / \mathrm{mL}$. Conclusions: Clinical algorithms using TDM might help to optimize the pharmacological therapy of IBD.

(Rev Med Chile 2018; 146: 1241-1251)

Key words: Crohn Disease; Ulcerative colitis; Drug Monitoring; Infliximab; Therapeutics.

I

nfliximab (IFX), un anticuerpo monoclonal IgG1 contra el factor de necrosis tumoral alfa (TNF- $\alpha$ ), ha demostrado ser efectivo en inducir y mantener la remisión en la Enfermedad Inflamatoria Intestinal (EII), incluyendo la Enfermedad de Crohn (EC) y la Colitis Ulcerosa $(\mathrm{CU})^{1-3}$. Sin embargo, la falta de respuesta pri-

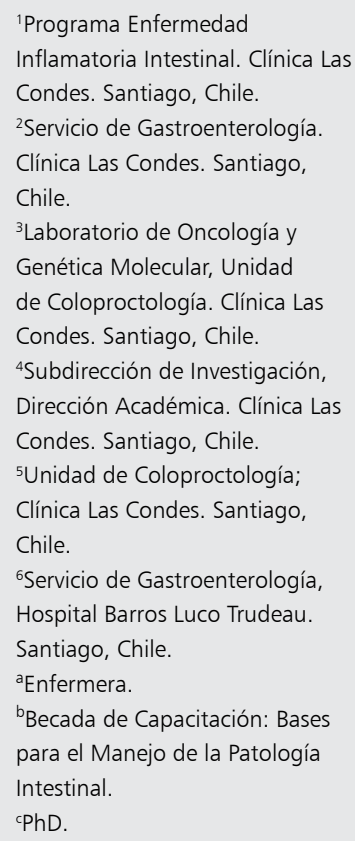

Fuente de Apoyo: Proyecto Clínica Las Condes N ${ }^{\circ}$ PIDA 2015-003.

Los autores declaran no tener conflictos de interés.

Recibido el 9 de abril de 2018, aceptado el 18 octubre de 2018.

Correspondencia a: Dr. Rodrigo Quera Clínica Las Condes. Estoril 450, Las Condes, Santiago, Chile. Teléfono 226108048. rquera@clc.cl maria (FRP) o la pérdida de respuesta secundaria (PRS) a éste y otros anti-TNF representa un desafió en muchos pacientes. La FRP se define como la ausencia de respuesta clínica post-inducción del anti-TNF y su frecuencia puede alcanzar hasta $40 \%{ }^{4-6}$. Por otra parte, la PRS se refiere al grupo de pacientes que inicialmente responden al esquema 
de inducción, pero que durante el tratamiento de mantención presentan una reactivación de la enfermedad. Estudios han determinado que su frecuencia varía entre $10-50 \%$ en un período de 52 semanas $^{7-9}$.

Los mecanismos asociados al desarrollo de estas dos condiciones incluyen factores farmacodinámicos y farmacocinéticos. El primero se relaciona con la presencia de vías inflamatorias diferentes a TNF- $\alpha^{6,10}$. Por otra parte, los mecanismos farmacocinéticos están relacionados con la presencia de concentraciones subterapéuticas o indetectables del fármaco, ya sea por una pérdida acelerada del medicamento por un mecanismo no autoinmune o por un mecanismo de inmunogenicidad (desarrollo de anticuerpos contra infliximab [ATI $])^{10-13}$. Estudios han demostrado que los niveles plasmáticos de ATI se correlacionan inversamente con los niveles del fármaco, una corta duración de su efectividad y con el riesgo de reacción post-infusión ${ }^{14}$.

La monitorización terapéutica del fármaco (MTF), definida como la evaluación de la concentración de la droga y de los niveles de anticuerpos contra éste, ha demostrado ser más efectiva que el manejo estándar en términos de resultados clínicos y costo efectividad, logrando de esta manera una terapia personalizada ${ }^{14-23}$. Pese a estos resultados, no existen publicaciones nacionales que hayan evaluado la monitorización de IFX y ATI y su efecto en pacientes con EII. El objetivo de este estudio es determinar la concentración de IFX y ATI en pacientes con EII tratados con este fármaco, evaluando el impacto en el curso clínico de la EII.

\section{Pacientes y Métodos}

Estudio observacional descriptivo de cohorte prospectiva realizado entre julio de 2016-mayo de 2017, donde se incluyeron pacientes diagnosticados con CU, EC o EII no-clasificable pertenecientes al Programa de EII de Clínica Las Condes, que iniciarían o se encontraban en tratamiento con IFX. La población en estudio se dividió en dos grupos. El Grupo A correspondió a pacientes vírgenes a tratamiento biológico que iniciaron IFX (5 o 10 $\mathrm{mg} / \mathrm{Kg}$ ) ya sea monoterapia o asociado a inmunomoduladores (azatioprina/6-mercaptopurina o metotrexato) en el período de estudio. El Grupo B incluyó a pacientes en tratamiento de mantención con IFX (al menos 4 dosis del fármaco).

Se excluyeron pacientes con CU fulminante, EC de fenotipo estenosante o fistulizante, o que hayan presentado reactivación de la enfermedad atribuido a sobreinfección por Clostridium difficile o Citomegalovirus. Las variables demográficas y clínicas fueron obtenidas del formulario de registro y seguimiento del Programa de EII.

\section{Determinación niveles de IFX y ATI}

La determinación de niveles de IFX y ATI se realizó a través del método ELISA según el protocolo definido por el fabricante (Infliximab ELISA Kit, ABIN1540247 y Anti-Infliximab Antibody ELISA Kit, ABIN1540248; EE.UU.). En el Grupo A, la medición de los niveles se realizó a la semana 14 y 30, y en el Grupo B fueron determinados previo a la administración de la siguiente dosis del biológico. Niveles de IFX en sangre entre 3-7 ug/ml fueron estimados como adecuados ${ }^{12}$, mientras que ATI $>10 \mathrm{AU} / \mathrm{ml}$ fueron considerados elevados.

\section{Determinación de la actividad inflamatoria}

La actividad clínica se determinó a través del índice de Mayo Clínico para CU ( $>2$ o índice individual $>1$ ) y el Harvey-Bradshaw $(>4)$ para $\mathrm{EC}^{24,25}$. Para el índice de actividad endoscópica se utilizó el índice de Mayo para CU, SES-CD para EC e índice de Rutgeerts para EC postcirugía ${ }^{24.26}$. Se definió como curación de mucosa $(\mathrm{CM})$, índice de Mayo 0-1 para CU y SES-CD 0-2 o Rutgeerts $\leq 2$ para $\mathrm{EC}^{27}$. En el grupo A, la colonoscopía fue realizada en la semana $14^{28,29} \mathrm{o}$ antes en caso de presentar actividad clínica o bioquímica. En el grupo B, se realizó colonoscopía en aquellos pacientes que presentaron actividad clínica y/o bioquímica. Esta última se estableció con calprotectina fecal (CF) $>250 \mathrm{ug} / \mathrm{g}^{30}$.

La estrategia terapéutica fue modificada de acuerdo a la presencia de actividad inflamatoria del paciente, demostrada por CF y/o colonoscopía, y niveles plasmáticos de IFX y ATI ${ }^{31,32}$.

Este estudio fue aprobado por el Comité de Ética de la institución y todos los pacientes firmaron un consentimiento informado previo a la inclusión en este estudio.

\section{Análisis estadístico}

Los datos fueron analizados mediante el programa $\mathrm{R}$ Commander. Las variables numéricas 
continuas se analizaron con mediana e intervalos, ya que no distribuían normal y se compararon mediante test de Mann Whitney. Para las variables cualitativas categóricas se utilizó frecuencia relativa porcentual y para el análisis estadístico comparativo $\chi^{2}$. Se determinó significancia estadística a un valor $\mathrm{p}<0,05$.

\section{Resultados}

Un total de 50 pacientes con EII tratados con IFX fueron incluidos, 14 pertenecientes al Grupo A y 36 al Grupo B. Las características demográficas y clínicas de cada grupo son presentadas en la Tabla 1. Hubo un paciente con diagnóstico

Tabla 1. Características clínicas y demográficas de los pacientes con Enfermedad Inflamatoria Intestinal al momento de ser ingresados al estudio

\begin{tabular}{|c|c|c|}
\hline & $\begin{array}{c}\text { Grupo } A \\
14 \text { pacientes }\end{array}$ & $\begin{array}{c}\text { Grupo B } \\
36 \text { pacientes }\end{array}$ \\
\hline Hombres (n; \%) & $7(50)$ & $24(66,7)$ \\
\hline Edad en años (mediana; rango) & $29(16-50)$ & $32(10-61)$ \\
\hline Años de enfermedad (mediana; rango) & $3(1-23)$ & $6,5(2-22)$ \\
\hline Albúmina (mediana; rango) & $4(3,7-4,9)$ & $4,2(3,4-4,8)$ \\
\hline IMC (mediana; rango) & $24,9(18,1-30,8)$ & $23,3(17,7-31)$ \\
\hline $\begin{array}{l}\text { Enfermedad de Crohn (n; \%) } \\
\text { Localización: } \\
\text { L1 } \\
\text { L2 } \\
\text { L3 } \\
\text { L1 + L4 } \\
\text { Fenotipo: } \\
\text { B1 } \\
\text { B2 } \\
\text { B3 } \\
\text { P }\end{array}$ & $\begin{array}{c}6(43) \\
2 \\
- \\
3 \\
1 \\
6 \\
- \\
- \\
-\end{array}$ & $\begin{array}{c}25(69,4) \\
6 \\
6 \\
12 \\
1 \\
23 \\
1 \\
1 \\
8\end{array}$ \\
\hline Antecedentes de cirugía (n; \%) & $1(7,1)$ & $7(19,4)$ \\
\hline $\begin{array}{l}\text { Índice endoscópico SES-CD: } \\
\text { Remisión }(0-2) \\
\text { Leve }(3-6) \\
\text { Moderado }(7-15) \\
\text { Severo }(\geq 16) \\
\text { Índice Rutgeerts: } \\
\text { i0 } \\
\text { i1 } \\
\text { i2 } \\
\text { i3 } \\
\text { i4 }\end{array}$ & $\begin{array}{l}- \\
- \\
4 \\
1 \\
- \\
- \\
- \\
- \\
1\end{array}$ & $\begin{array}{l}7 \\
2 \\
3 \\
- \\
4 \\
- \\
- \\
1 \\
2\end{array}$ \\
\hline $\begin{array}{l}\text { Colitis ulcerosa (n; \%) } \\
\text { E1 } \\
\text { E2 } \\
\text { E3 }\end{array}$ & $\begin{array}{c}8(57,1) \\
- \\
2 \\
6\end{array}$ & $\begin{array}{c}10(27,7) \\
- \\
3 \\
7\end{array}$ \\
\hline $\begin{array}{l}\text { Índice endoscópico Mayo } \\
\text { Mayo } 0 \\
\text { Mayo } 1 \\
\text { Mayo } 2 \\
\text { Mayo } 3\end{array}$ & $\begin{array}{l}- \\
- \\
4 \\
4\end{array}$ & $\begin{array}{l}4 \\
2 \\
2 \\
-\end{array}$ \\
\hline Calprotectina fecal (ug/g) (mediana; rango) & $600(15-1.380)$ & $102(10-1.619)$ \\
\hline $\begin{array}{l}\text { Tratamiento asociado a Infliximab: } \\
\text { Tiopurínicos (azatioprina/6-mercaptopurina) } \\
\text { Metotrexato } \\
\text { Esteroides (prednisona/budesonida) } \\
\text { Mesalazina }\end{array}$ & $\begin{array}{l}6 \\
7 \\
3 \\
3\end{array}$ & $\begin{array}{r}21 \\
9 \\
- \\
5\end{array}$ \\
\hline
\end{tabular}

IMC: índice de masa corporal. 
de EII no-clasificable. En el grupo A, 11/14 pacientes tuvieron una evaluación de la actividad inflamatoria con colonoscopía y CF y tres sólo con colonoscopía. En el grupo B, 19/36 pacientes fueron evaluados por colonoscopía y CF, 10 sólo con CF y 7 sólo colonoscopía.

\section{Grupo A}

$57,1 \%$ (8/14 pacientes) alcanzaron CM después de completar la fase de inducción. La mediana de los niveles de IFX a la semana 14 fue de 2,65 AU/ $\mathrm{mL}(0,3-32,58)$ con ocho pacientes presentando niveles $<3 \mathrm{AU} / \mathrm{mL}$, cuatro con niveles terapéuticos entre 3-7 AU/mL y dos con niveles $>7 \mathrm{AU} /$ $\mathrm{mL}$. Al evaluar las concentraciones de IFX, éstas fueron mayores en los pacientes que alcanzaron $\mathrm{CM}$, sin significancia estadística (mediana 3,2 vs $2,2 ; \mathrm{p}=0,6)$ (Figura 1). Solo un paciente tuvo ATI $>10 \mathrm{ug} / \mathrm{mL}$, teniendo además niveles de IFX de $0,3 \mathrm{AU} / \mathrm{mL}$, quien se encontraba en tratamiento con metotrexato.

En los seis pacientes que presentaron una falta de respuesta a IFX, la estrategia fue aumentar la dosis a $10 \mathrm{mg} / \mathrm{Kg}$. Sin embargo, uno de ellos requirió cirugía al no tener una respuesta clínica al completar la inducción con $10 \mathrm{mg} / \mathrm{Kg}$, con un nivel plasmático post dosis de $45 \mathrm{AU} / \mathrm{mL}$.

De los 13 pacientes que tuvieron una segunda medición a la semana 30 , la mediana de los niveles de IFX fue de $2,3 \mathrm{AU} / \mathrm{mL}(0,3-16,8)$ con nueve pacientes presentando niveles $<3 \mathrm{AU} / \mathrm{mL}$, tres con niveles terapéuticos entre 3-7 AU/mL $\mathrm{y}$ uno con niveles $>7 \mathrm{AU} / \mathrm{mL}$. Siete pacientes, de los cuales seis estaban con inmunosupresor, tuvieron ATI $>10 \mathrm{ug} / \mathrm{mL}$ (13-174), todos ellos con niveles de IFX $<3 \mathrm{AU} / \mathrm{mL}(0,3-2,3)$. Dos de ellos estaban activos clínicamente y por CF por lo que en un paciente se optimizo dosis de IFX a 10 $\mathrm{mg} / \mathrm{Kg}$ y en el segundo se insistió en adherencia a inmunosupresor.

Al seguimiento de 12 meses (Figura 2), 12 de los 13 pacientes que iniciaron un esquema de mantención mantenían el tratamiento con IFX. Cuatro pacientes $(30,7 \%)$ presentaron PRS durante este período, demostrado por actividad clínica y endoscópica. La mediana de los niveles

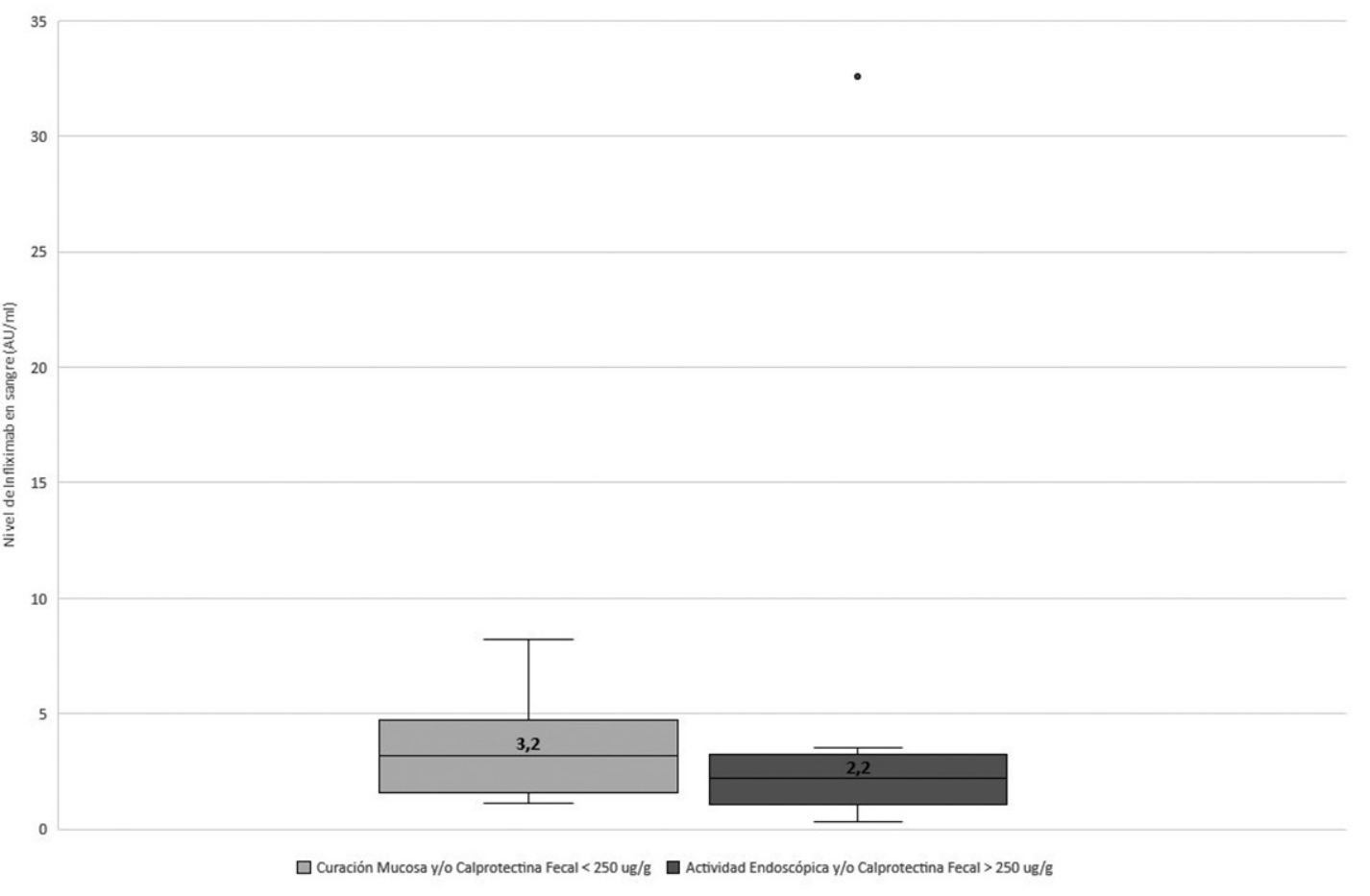

Figura 1. 


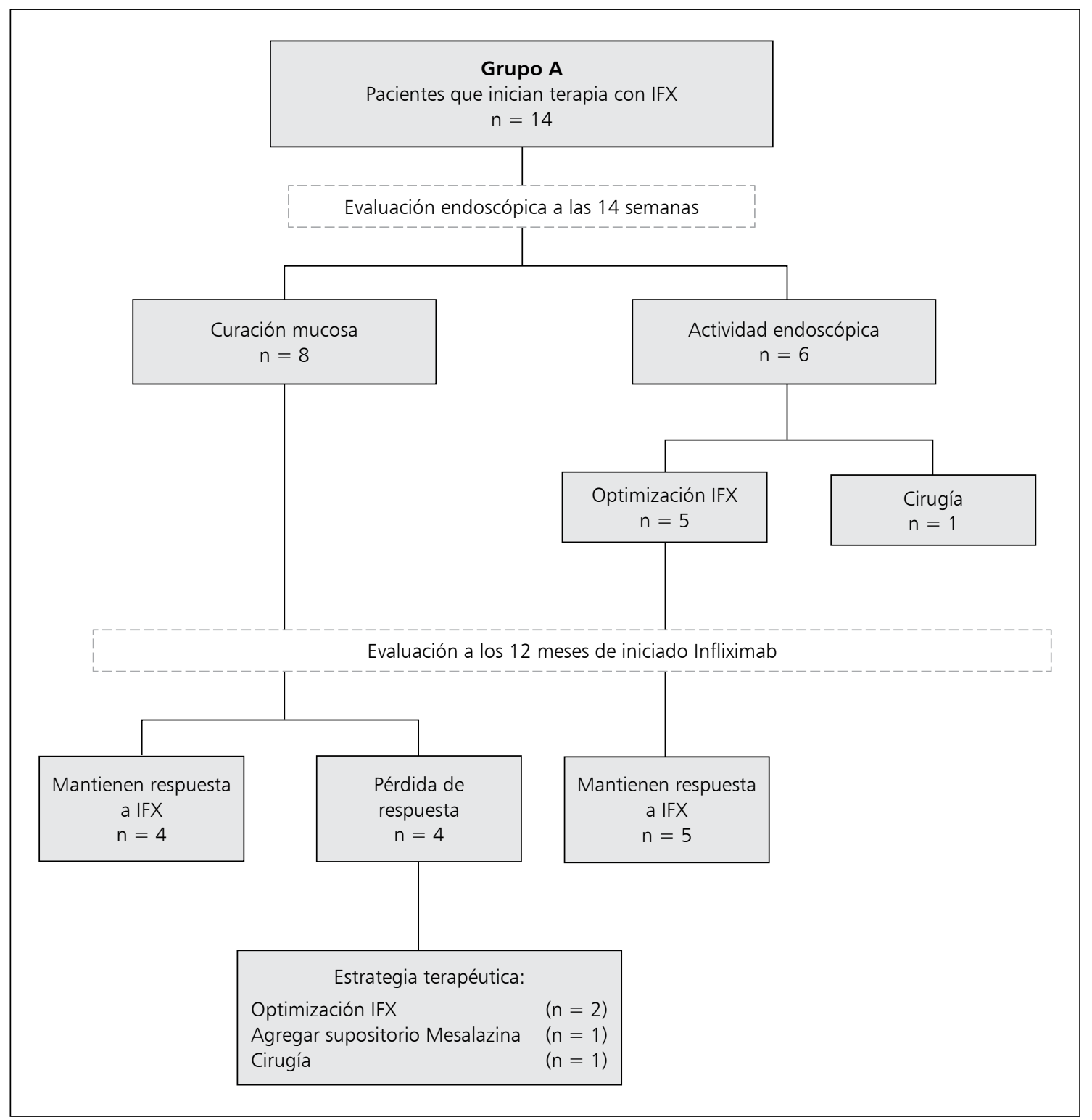

Figura 2. Seguimiento de un año de pacientes que iniciaron tratamiento con Infliximab (IFX).

de IFX y ATI en estos pacientes fue de 2,65 AU/ $\mathrm{mL}(1,6-4,5)$ y $6,6 \mathrm{ug} / \mathrm{mL}$ (2-13), respectivamente. La estrategia terapéutica fue optimizar la dosis de IFX a $10 \mathrm{mg} / \mathrm{Kg}$ en tres pacientes y agregar mesalazina supositorio en un paciente con CU con actividad endoscópica Mayo 2 en el recto. Uno de los tres pacientes no respondió a la optimización de IFX, requiriendo cirugía debido a la presencia de estenosis con componente inflamatorio a nivel de colon (niveles de IFX 2,5 AU/mL y ATI 8,4 ug/ $\mathrm{mL}$ ). Los otros pacientes presentaron una respuesta clínica teniendo una $\mathrm{CF}<250 \mathrm{ug} / \mathrm{g}$.

\section{Grupo B}

Al momento de la medición, 24 (66,7\%) de los 36 pacientes se encontraban en remisión clínica con $\mathrm{CF}<250 \mathrm{ug} / \mathrm{g}$ y/o CM en la colonoscopía. La mediana de infusiones de IFX al momento de 


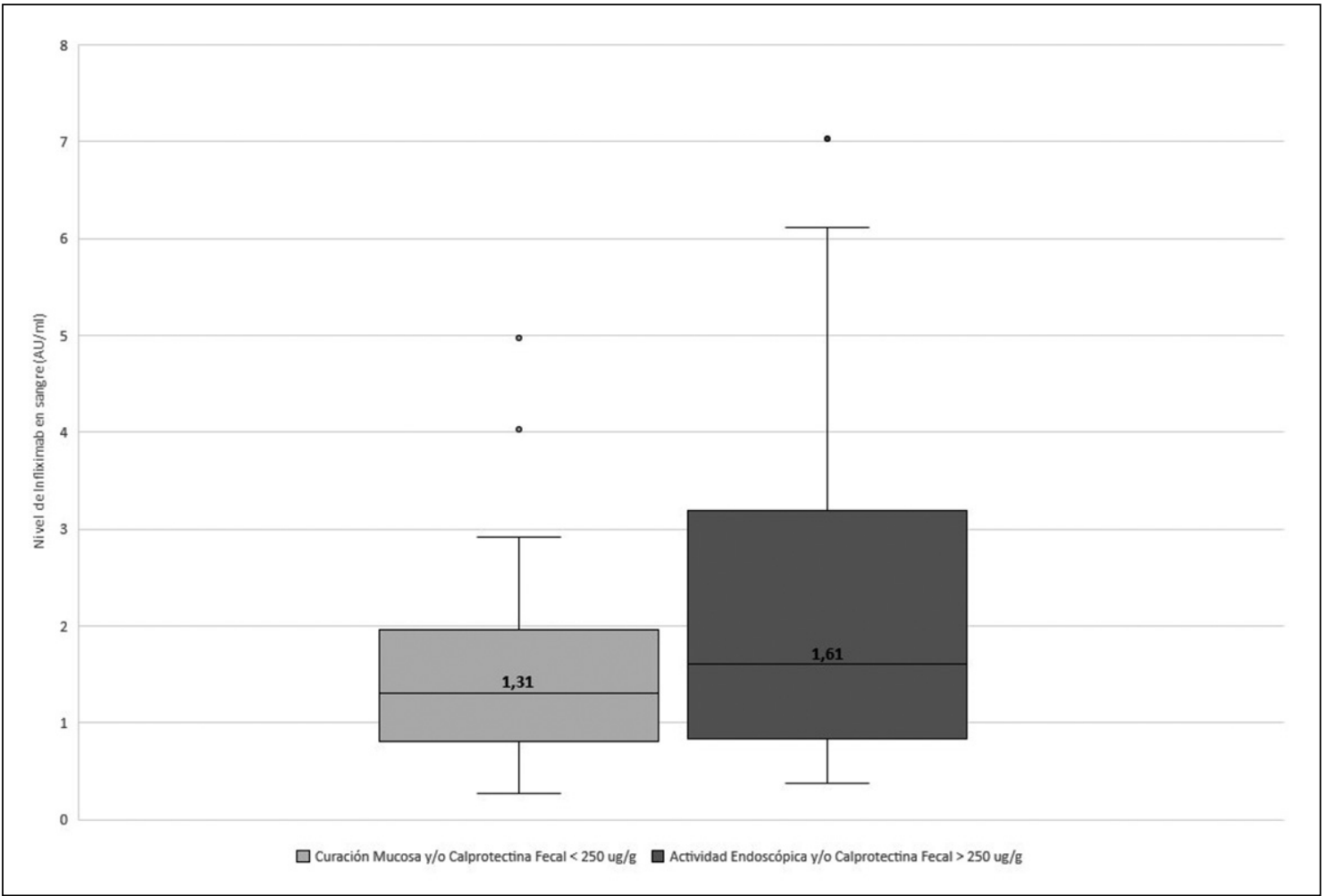

Figura 3.

la medición fue de 14 (4-96). La mediana de los niveles del fármaco fue de $1,4 \mathrm{AU} / \mathrm{mL}(0,27-7,03)$ con 31 pacientes presentando niveles $<3 \mathrm{AU} / \mathrm{mL}$, cuatro con niveles terapéuticos entre 3-7 AU/ML y uno con niveles $>7 \mathrm{AU} / \mathrm{mL}$. Los niveles de IFX no fueron significativamente mayores en aquellos pacientes que estaban en remisión al momento de la evaluación en comparación a aquellos con actividad inflamatoria (mediana $1,61 \mathrm{AU} / \mathrm{mL}$ vs $1,31 \mathrm{AU} / \mathrm{mL} ; \mathrm{p}=0,99$ ) (Figura 3). Diecisiete pacientes, 13 de ellos con inmunosupresor, tuvieron ATI $>10 \mathrm{ug} / \mathrm{mL}$.

En los 12 pacientes que presentaron actividad inflamatoria al momento de la evaluación, la estrategia terapéutica fue cambiar de biológico en cuatro pacientes, optimizar la dosis de IFX a $10 \mathrm{mg} / \mathrm{Kg}$ en tres pacientes, optimizar la dosis de inmunosupresores en tres pacientes y cirugía en dos pacientes.

Al seguimiento a los 12 meses de la medición (Figura 4), seis pacientes presentaron actividad inflamatoria siendo necesario un cambio de estrategia terapéutica con optimización de adalimumab en dos pacientes, cambio de biológico por PRS a IFX en un paciente, optimización de IFX a $10 \mathrm{mg} / \mathrm{Kg}$ en un paciente, inicio de adalimumab por reaparición de actividad inflamatoria posterior a suspensión de IFX (niveles de IFX $<1$ AU/mL, ATI 471,78 ug/mL y colonoscopía con remisión endoscópica e histológica previo a la suspensión) en un paciente y cirugía por imposibilidad de cobertura para cambio de biológico en un paciente.

\section{Discusión}

A nivel nacional, IFX es el biológico más utilizado en el manejo de pacientes con EII. Hasta hace poco tiempo, el manejo estándar de la FRP y la PRS era modificar de manera empírica el tratamiento, ya sea optimizando el fármaco (aumentando la dosis y/o acortando la frecuencia de administración), o cambiando a otro anti-TNF u 


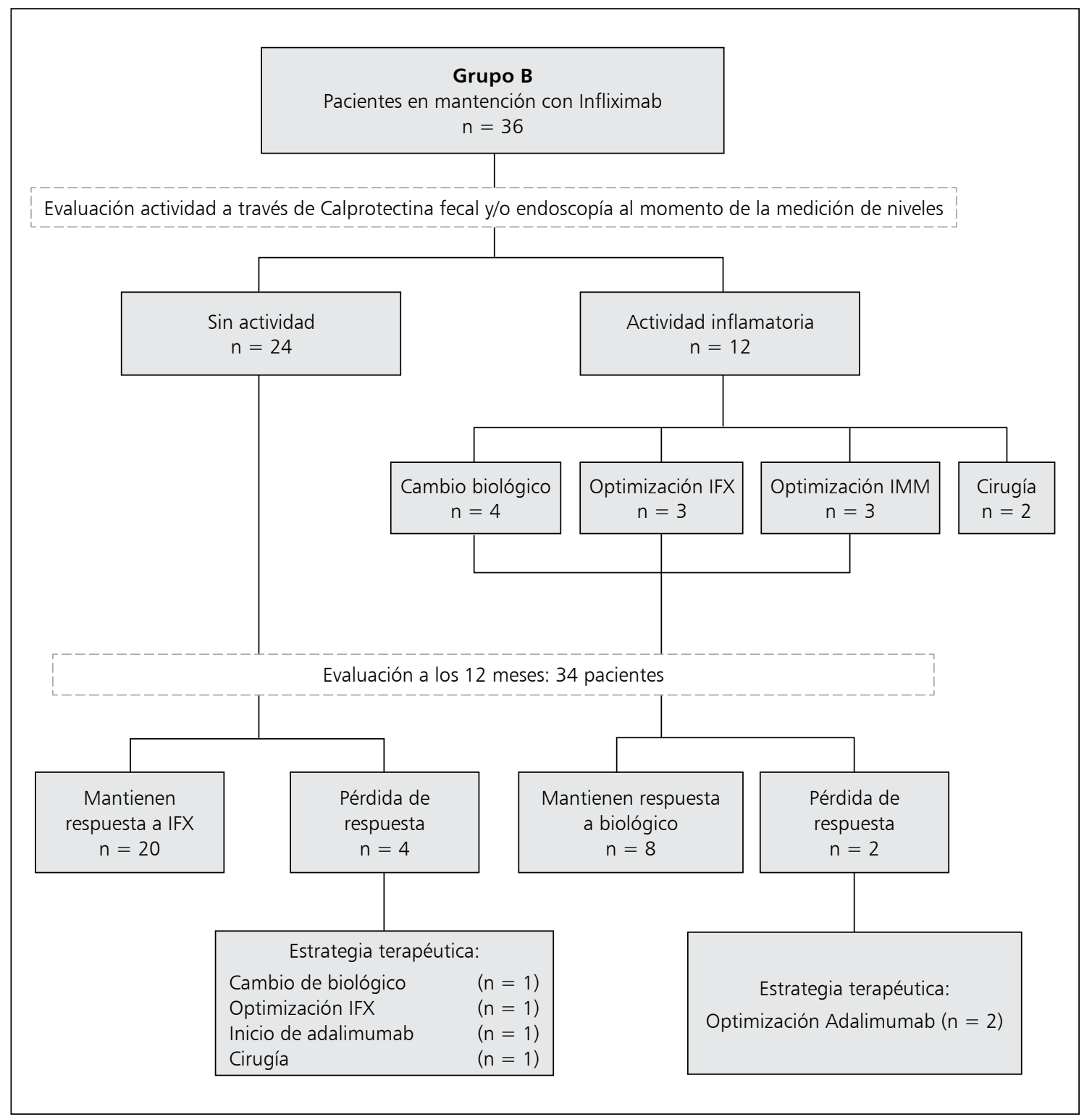

Figura 4. Seguimiento de un año de pacientes en terapia de mantención con Infliximab Grupo B.

otro biológico con diferente mecanismo de acción. Sin embargo, estas estrategias, al no considerar aspectos de la farmacocinética o farmacodinámica del anti-TNF, pueden conducir a una exposición innecesaria al fármaco, un potencial aumento en los costos del tratamiento, y eventual desarrollo de eventos adversos ${ }^{33}$. Esta es la primera publicación nacional que muestra el uso de la MTF en el manejo de los pacientes con EII. Esta herramienta permitiría definir de mejor forma una estrategia terapéutica diferente frente a la falta o pérdida de respuesta, logrando con ello un manejo cada vez más personalizado ${ }^{34}$. Estudios han demostrado igualmente la utilidad de la monitorización de otros anti-TNF como adalimumab ${ }^{35}$ o golimumab $^{36}$ y biológicos con otros mecanismos de acción como vedolizumab ${ }^{37}$.

Un estudio reciente muestra que los gastroen- 
terólogos subutilizan la MTF, no por la falta de estudios que avalen su utilidad, sino por los costos económicos que implica su uso ${ }^{38}$. Sin embargo, independientemente de esto, los pacientes se han mostrado proclives a esta estrategia de modo de tener un tratamiento más personalizado ${ }^{39}$.

Aunque el nivel terapéutico de IFX no ha sido idéntico entre los diferentes estudios, niveles entre 3-7 ug/mL han sido correlacionados de manera positiva con la posibilidad de alcanzar remisión clínica y endoscópica ${ }^{20}$. Por otra parte, la presencia de ATI ha sido asociada a una pérdida de respuesta a esta terapia ${ }^{40,41}$. Sin embargo, la interpretación de los ATI y otros biológicos debe ser realizada con precaución dado que su concentración puede ser fluctuante e incluso desaparecer con la intensificación del biológico ${ }^{42}$. Varios ensayos han sido utilizados para evaluar la concentración de IFX y la presencia de anticuerpos, destacando el enzimo inmunoanálisis de absorción (ELISA), ensayo por movilidad homogénea (HMSA) y el inmunoensayo electroquimioluminicencia (ECLIA). Hasta ahora, ninguno de ellos ha sido considerado el gold estándar, sugiriéndose que estos métodos de medición no deben ser intercambiables y que la comparación entre niveles obtenidos de diferentes ensayos debe ser evitado ${ }^{43}$. En nuestro estudio, utilizamos un método de ELISA con la capacidad de medir la presencia de anticuerpos totales, es decir anticuerpos circulantes y unidos al fármaco.

Es importante considerar que la MTF es dinámica, dependiendo de la etapa de intervención (inducción vs mantención), objetivos del tratamiento (remisión clínica vs endoscópica vs histológica) y actividad de la enfermedad (enfermedad luminal vs perianal ${ }^{44,45}$. En este último escenario, Yarur y cols. demostraron recientemente que niveles de IFX $>10,1 \mathrm{AU} / \mathrm{mL}$ se asocian a una mayor probabilidad de curación de la fístula ${ }^{46}$. En nuestro estudio, aunque los ocho pacientes con enfermedad perianal presentaron niveles de IFX bajo este rango, solo dos de ellos tenían actividad de la enfermedad decidiéndose aumentar la dosis de IFX al tener ATI $<10 \mathrm{ug} / \mathrm{mL}$.

En pacientes que inician terapia biológica, varios estudios han demostrado el impacto de la MTF a corto y largo plazo ${ }^{17,21,47-51}$. En el Grupo A, de los seis pacientes que presentaron actividad inflamatoria antes de la $4^{\text {a }}$ dosis de IFX, solo uno no respondió a la estrategia de optimizar la dosis de IFX siendo necesario realizar una colectomía total. Esta paciente ya tenía niveles de IFX $>7$ AU/ $\mathrm{mL}$ antes de optimizar la dosis, confirmando que factores farmacodinámicos explicarían la refractariedad a este biológico ${ }^{31,32}$. Estudios han señalado que pacientes con niveles $>3 \mathrm{AU} / \mathrm{mL}$ presentan mejores resultados terapéuticos que pacientes bajo este rango ${ }^{17,51}$. El reducido número de pacientes incluido en este grupo no permitió analizar estos resultados.

En pacientes en tratamiento con IFX en esquema de mantención, dos estrategias han sido sugeridas en la práctica clínica ${ }^{18,23,32-56}$. Por una parte, la MTF ha sido planteada cuando los pacientes presentan evidencias de actividad inflamatoria, lo que permite definir la mejor estrategia terapéutica siendo esta decisión más costo efectiva que escalar de manera empírica ${ }^{31,32}$. En nuestro estudio, 12/36 pacientes $(33,3 \%)$ del Grupo B presentaban actividad inflamatoria al momento de la MTF siendo posible con los niveles de IFX y ATI individualizar la terapia. En el Grupo A, cuatro de los 13 pacientes que recibieron más de 3 dosis de IFX presentaron PRS durante el primer año de tratamiento siendo necesario modificar su tratamiento. Por otra parte, recientemente se ha demostrado que una estrategia proactiva con titulación de la dosis del biológico con el objetivo de obtener concentraciones predeterminadas, podría mejorar los resultados clínicos al aumentar la duración de la efectividad del fármaco y disminuir la necesidad de cirugía y hospitalización asociada a la EII ${ }^{18,23,56}$. Además, estudios han evaluado el uso de esta estrategia con el fin de definir la probabilidad de recaída al suspender el tratamiento ${ }^{20,57,58}$. Niveles de IFX $>4,5 \mathrm{ug} / \mathrm{mL}$ al momento de retirar el fármaco sería predictivo de recaída. En el Grupo B, de los 24 pacientes que se encontraban sin actividad inflamatoria, solo se realizó cambio en el tratamiento en una paciente quien se encontraba en remisión profunda después de cuatro años con IFX. Este fármaco se suspendió al tener niveles $<1 \mathrm{AU} / \mathrm{mL}$ y ATI 471,78 ug/mL. Estudios prospectivos deberán confirmar la efectividad de la estrategia proactiva en pacientes que se encuentran en tratamiento de mantención con biológicos ${ }^{59}$.

Estudios han demostrado el beneficio de los inmunomoduladores en la farmacocinética e inmunogenicidad del IFX ${ }^{51,60,61}$. En nuestro estudio, 13 de los 14 pacientes del Grupo A y 30 de los 36 del Grupo B se encontraban en tratamiento con inmunomoduladores, por lo que no fue posible 
evaluar el efecto del uso de tiopurínicos/metotrexato en los niveles de IFX y ATI.

En conclusión, nuestro estudio muestra que la MTF podría tener un rol en el manejo de pacientes con EII. Estudios prospectivos a nivel local podrán determinar la efectividad de esta estrategia en otros escenarios y con otros anti-TNF. Sin duda estos resultados permitirán personalizar el tratamiento de cada paciente de manera efectiva y segura.

\section{Referencias}

1. Simian D, Quijada MI, Lubascher J, Acuña R, Quera R. Treatment of Inflammatory Bowel Disease with infliximab: experience in 25 patients. Rev Med Chile 2013; 141: 1158-65.

2. Mao EJ, Hazlewood GS, Kaplan GG, Peyrin-Biroulet L, Ananthakrishnan AN. Systematic review with meta-analysis: comparative efficacy of immunosuppressants and biologics for reducing hospitalization and surgery in Crohn's disease and ulcerative colitis. Aliment Pharmacol Ther 2017; 45: 3-13.

3. Cholapranee A, Hazlewood GS, Kaplan GG, Peyrin-Biroulet L, Ananthakrishnan AN. Systematic review with meta-analysis: comparative efficacy of biologics for induction and maintenance of mucosal healing in Crohn's disease and ulcerative colitis controlled trials. Aliment Pharmacol Ther 2017; 45: 1291-302.

4. Allez M, Karmiris K, Louis E. Report of the ECCO pathogenesis workshop on anti-TNF therapy failures in inflammatory bowel disease: definitions, frequency and pharmacological aspects. J Crohns Colitis 2010; 4: 355-66.

5. Ben-Horin S, Chowers Y. Tailoring anti-TNF therapy in IBD: drug levels and disease activity. Nat Rev Gastroenterol Hepatol 2014; 11: 243-55.

6. Papamichel K, Gils A, Rutgeerts P, Levesque BG, Vermeire S, Sandborn WJ, et al. Role for therapeutic drug monitoring during induction therapy with TNF antagonists in IBD: evolution in the definition and management of primary nonresponse. Inflamm Bowel Dis 2015; 21: 182-97.

7. Roda G, Jharap B, Neeraj N, Colombel JF. Loss of response to anti-TNFs: definition, epidemiology, and management. Clin Transl Gastroenterol 2016; 7: e135.

8. Gisbert JP, Panes J. Loss of response and requirement of infliximab dose intensification in Crohn's disease: a review. Am J Gastroenterol 2009; 104: 760-7.

9. Ben-Horin S, Chowers Y. Review article: loss of response to anti-TNF treatments in Crohn's disease. Aliment
Pharmacol Ther 2011; 33: 987-95.

10. Ben-Horin S, Kopylov U, Chowers Y. Optimizing anti-TNF treatments in inflammatory bowel disease. Autoimmun Rev 2014; 13: 24-30.

11. Nanda KS, Cheifetz AS, Moss AC. Impact of antibodies to infliximab on clinical outcomes and serum infliximab levels in patients with inflammatory bowel disease (IBD): a meta-analysis. Am J Gastroenterol 2013; 108: 40-7.

12. Vande Casteele N, Khanna R, Levesque BG, Stitt L, Zou GY, Singh S, et al. The relationship between infliximab concentrations, antibodies to infliximab and disease activity in Crohn's disease. Gut 2015; 64: 1539-45.

13. Vande Casteele N, Gils A. Pharmacokinetics of anti-TNF monoclonal antibodies in inflammatory bowel disease: adding value to current practice. J Clin Pharmacol 2015; 55 (suppl 3): S39-S50.

14. Adedokun OJ, Sandborn WJ, Feagan BG, Rutgeerts P, $\mathrm{Xu} \mathrm{Z}$, Marano CW, et al. Association between serum concentration of infliximab and efficacy in adult patients with ulcerative colitis. Gastroenterology 2014; 147: 1296-307.

15. Velayos FS, Kahn JG, Sandborn WJ, Feagan BG. A test-based strategy is more cost effective than empiric dose escalation for patients with Crohn's disease who lose responsiveness to infliximab. Clin Gastroenterol Hepatol 2013; 11: 654-66.

16. Steenholdt C, Brynskov J, Thomsen OØ, Munck LK, Fallingborg J, Christensen LA, et al. Individualised therapy is more cost-effective than dose intensification in patients with Crohn's disease who lose response to anti-TNF treatment: a randomised, controlled trial. Gut 2014; 63: 919-27.

17. Cornillie F, Hanauer SB, Diamond RH, Wang J, Tang $\mathrm{KL}, \mathrm{Xu} Z$, et al. Postinduction serum infliximab trough concentration and decrease of C-reactive protein concentration are associated with durable sustained response to infliximab: a retrospective analysis of the ACCENT I trial. Gut 2014; 63: 1721-27.

18. Vaughn BP, Martínez-Vázquez M, Patwardhan VR, Moss AC, Sandborn WJ, Cheifetz AS. Proactive therapeutic concentration monitoring of infliximab may improve outcomes for patients with inflammatory bowel disease: results from a pilot observational study. Inflamm Bowel Dis 2014; 20: 1996-2003.

19. Yanai H, Lichtenstein L, Assa A, Mazor Y, Weiss B, Levine $\mathrm{A}$, et al. Levels of drug and antidrug antibodies are associated with outcome of interventions after loss of response to infliximab or adalimumab. Clin Gastroenterol Hepatol 2015; 13: 522-30.

20. Vande Casteele N, Ferrante M, Van Assche G, Ballet 
V, Compernolle G, Van Steen K, et al. Trough concentrations of infliximab guide dosing for patients with inflammatory bowel disease. Gastroenterology 2015; 148: 1320-9.

21. Papamichael K, Van Stappen T, Vande Casteele N, Gils A, Billiet T, Tops S, et al. Infliximab concentration thresholds during induction therapy are associated with short-term mucosal healing in patients with ulcerative colitis. Clin Gastroenterol Hepatol 2016; 14: 543-9.

22. Billiet T, Cleynen I, Ballet V, Ferrante M, Van Assche G, Gils A, et al. Prognostic factors for long-term infliximab treatment in Crohn's disease patients: a 20-year single centre experience. Aliment Pharmacol Ther 2016; 44: 673-83.

23. Papamichael K, Chachu KA, Vajravelu RK, Vaughn BP, Ni J, Osterman MT, et al. Improved long-term outcomes of patients with inflammatory bowel disease receiving proactive compared with reactive monitoring of serum concentrations of infliximab. Clin Gastroenterol Hepatol 2017; 15: 1580-8.

24. Schroeder KW, Tremaine WJ, Ilstrup DM. Coated oral 5-aminosalicylic acid therapy for mildly to moderately active ulcerative colitis. A randomized study. N Engl J Med 1987; 317: 1625-9.

25. Best WR, Becktel JM, Singleton JW, Kern F Jr. Development of a Crohn's disease activity index. National Cooperative Crohn's Disease Study. Gastroenterology 1976; 70: 439-44.

26. Vuitton L, Marteau P, Sandborn WJ, Levesque BG, Feagan B, Vermeire S, et al. IOBD technical review on endoscopic indices for Corhn's disease clinical trials. Gut 2016; 65: 1477-55.

27. Flortholmen J. Mucosal healing in the era of biological agents in treatment of Inflammatory Bowel Disease. Scand J Gastroenterol 2015; 50: 43-52.

28. af Björkesten CG, Nieminen U, Sipponen T, Turunen $\mathrm{U}$, Arkkila P, Färkkilä M. Mucosal healing at 3 months predict long-term endoscopic remission in anti-TNF treated luminal Crohn's disease. Scand J Gastroenterol 2013; 48: 543-51.

29. Figueroa C, Lubascher J, Ibañez J, Quera R, Kronberg U, Simian D, et al. Algoritmos de tratamiento de la Colitis Ulcerosa desde una experiencia local. Rev Med Clin Condes 2018; 29: 570-9.

30. Lin JF, Chen JM, Zuo JH, Yu A, Xiao ZJ, Deng FH, et al. Meta-analysis: fecal calprotectin for assessment of inflammatory bowel disease. Inflamm Bowel Dis 2014; 20: 1407-15.

31. Vande Casteele N, Herfarth H, Katz J, Falck-Ytter Y, Singh S. American Gastroenterological Association Technical review on the role of therapeutic drug monito- ring in the management of inflammatory bowel disease. Gastroenterology 2017; 153: 835-57.

32. Mitrev N, Vande Casteele N, Seow CH, Andrews JM, Connor SJ, Moore GT, et al. Review article: consensus statements on therapeutic drug monitoring of anti-tumor necrosis factor therapy in inflammatory bowel disease. Aliment Pharmacol Ther 2017; 46: 1037-53.

33. Colombel JF, Narula N, Peyrin-Biroulet L. Management strategies to improve outcomes of patients with inflammatory bowel diseases. Gastroenterology 2017; 152: 351-61.

34. Flamant M, Roblin X. Inflammatory bowel disease: towards a personalized medicine. Ther Adv Gastroenterol 2018 Jan 10.

35. Gonczi L, Kurti Z, Rutka M, Vegh Z, Farkas K, LovasZ $\mathrm{BD}$, et al. Drug persistence and need for dose intensification to adalimumab therapy; the importance of therapeutic drug monitoring in inflammatory bowel diseases. BMC Gastroenterol 2017; 17: 97.

36. Sheridan J, Coe CA, Doran P, Egan L, Cullen G, Kevans $\mathrm{D}$, et al. Protocol for a multicentre randomised controlled trial investigating the use of personalised golimumab dosing tailored to inflammatory load in ulcerative colitis: the GOAL-ARC study (GLM dose optimization to adequate levels to achieve response in colitis) led by the INITIAtive group (NCT 0268772). BMJ Open Gastroenterol 2018; 5: e000174.

37. Yacoub W, Williet N, Pouillon L, Di-Bernado T, De Carvalho Bittencouurt M, Nancey S, et al. Early vedolizumab trough levels predict mucosal healing in inflammatory bowel disease: a multicenter prospective observational study. Aliment Pharmacol Ther 2018; 47: 906-12.

38. Grossberg LB, Papamichael K, Feuerstein JD, Siegel CA, Ullman TA, Cheifetz AS. A survey study of gastroenterologists' attitudes and barriers toward therapeutic drug monitoring of anti-TNF therapy in Inflammatory Bowel Disease. Inflamm Bowel Dis 2017; 24: 191-7.

39. Campbell JP, Burton E, Wymer S, Shaw M, Vaughn BP. Out-of-pocket cost is a barrier to therapeutic drug monitoring in inflammatory bowel disease. Dig Dis Sci 2017; 62: 3336-43.

40. Hanauer SB, Wagner CL, Bala M, Mayer L, Travers $\mathrm{S}$, Diamond $\mathrm{RH}$, et al. Incidence and importance of antibody responses to infliximab after maintenance or episodic treatment in Crohn's disease. Clin Gastroenterol Hepatol 2004; 2: 542-53.

41. Nanda KS, Cheifetz AS, Moss AC. Impact of antibodies to infliximab on clinical outcomes and serum infliximab level in patients with inflammatory bowel disease: a meta-analysis. Am J Gastroenterol 2013; 108: 40-7. 
42. Colombel JF, Feagan BG, Sandborn WJ, Van Assche G, Robinson AM. Therapeutic drug monitoring of biologics for inflammatory bowel disease. Inflamm Bowel Dis 2012; 18: 349-58.

43. Bader LI, Solberg SM, Kaada SH, Bolstad N, Warren DJ, Gavasso S, et al. Assays for infliximab drug levels and antibodies: A matter of scales and categories. Scand J Immunol 2017; 86: 165-70.

44. Roblin X, Boschetti G, Duru G, Williet N, Deltedesco E, Phelip JM, Peyrin-Biroulet L, et al. Distinct thresholds of infliximab trough levels are associated with different therapeutic outcomes in patients with Inflammatory Bowel Disease: A prospective observational study. Inflamm Bowel Dis 2017; 23: 2048-53.

45. Papamichael K, Rakowsky S, Rivera C, Cheifetz AS, Osterman MT. Infliximab trough concentrations during maintenance therapy are associated with endoscopic and histologic healing in ulcerative colitis. Aliment Pharmacol Ther 2018; 47: 478-84.

46. Yarur AJ, Kanagala V, Stein DJ, Czul F, Quintero MA, Agrawal D, et al. Higher infliximab trough levels are associated with perianal fistula healing in patients with Crohn's disease. Aliment Pharmacol Ther 2017; 45: 93340.

47. Vande Casteele N, Gils A, Singh S, Ohrmund L, Hauenstein S, Rutgeerts $\mathrm{P}$, et al. Antibody response to infliximab and its impact on pharmacokinetics can be transient. Am J Gastroenterol 2013; 108: 962-71.

48. Arias MT, Vande Casteele N, Vermeire S, de Buck van Overstraeten A, Billiet T, Baert F, et al. A panel to predict long-yherm outcome of infliximab therapy for patients with ulcerative colitis. Clin Gastroenterol Hepatol 2015; 13: 531-8.

49. Van Stappen T, Bollen L, Vande Casteele N, Papamichael K, Van Assche G, Ferrante M, et al. Rapid test for infliximab drug concentration allows immediate dose adaptation. Clin Transl Gastroenterol 2016; 7: e206.

50. Hibi T, Sakuraba A, Watanabe M, Motoya S, Ito H, Sato N, et al. C-reactive protein is an indicator of serum infliximab level in predicting loss of response in patients with Crohn's disease. J Gastroenterol 2014; 49: 254-62.

51. Bortlik M, Duricova D, Malickova K, Machkova N, Bouzkova E, Hrdlicka L, et al. Infliximab trough levels may predict sustained response to infliximab in patients with Crohn's disease. J Crohns Colitis 2013; 7: 736-43.

52. Maser EA, Villela R, Silverberg MS, Greenberg GR. Association of trough serum infliximab to clinical outcome after scheduled maintenance treatment for Crohn's disease. Clin Gastroenterol Hepatol 2006; 4: 1248-54.

53. Ungar B, Levy I, Yavne Y, Yavzori M, Picard O, Fudim E, et al. Optimizing anti-TNF-a therapy: Serum levels of infliximab and adalimumab are associated with mucosal healing in patients with Inflammatory Bowel Disease. Clin Gastroenterol Hepatol 2016; 14: 550-7.

54. Imaeda H, Bamba S, Takakashi K, Fujimoto T, Ban H, Tsujikawa $\mathrm{T}$, et al. Relationship between serum infliximab trough levels and endoscopic activities in patients with Crohn's disease under scheduled maintenance treatment. J Gastroenterol 2014; 49: 674-82.

55. Warman A, Straathof JW, Derijks LJJ. Therapeutic drug monitoring of infliximab in inflammatory bowel disease patients in a teaching hospital setting results of a prospective cohort study. Eur J Gastroenterol Hepatol 2015; 27: 242-8.

56. Papamichael K, Vajravelu RK, Vaughn BP, Osterman MT, Cheifetz AS. Proactive infliximab monitoring following reactive testing is associated with better clinical outcomes than reactive testing alone in patients with inflammatory bowel disease. J Crohn Colitis 2018 Mar 24.

57. Louis E, Mary JY, Vernier-Massouille G, Grimaud JC, Bouhnik Y, Laharie D, et al. Maintenance of remission among patients with Crohn's disease on metabolite therapy after infliximab therapy is stopped. Gastroenterology 2012; 142: 63-70.

58. Ben-Horin S, Chowers Y, Ungar B, Kopylov U, Loebstein R, Weiss B, et al. Undetectable anti-TNF drug levels in patients with long-therm remission predict successful drug withdrawal. Aliment Pharmacol Ther 2015; 42: 356-64.

59. Ricciuto A, Dhaliwal J, Walters TD, Griffiths AM, Church PC. Clinical outcomes with therapeutic drug monitoring in Inflammatory Bowel Disease: A systematic review with meta-analysis. J Crohns Colitis 2018, Aug 10.

60. Feagan BG, McDonald JW, Panaccione R, Enns RA, Bernstein CN, Ponich TP, et al. Methotrexate in combination with infliximab is no more effective than infliximab alone in patients with Crohn's disease. Gastroenterology 2014; 146: 681-8.

61. Christophorou D, Funakoshi N, Duny Y, Valats JC, Bismuth M, Pineton De Chambrun G, et al. Systematic review with meta-analysis: infliximab and immunosuppressant therapy vs infliximab alone for active ulcerative colitis. Aliment Pharmacol Ther 2015; 41: 603-12. 